\title{
Aspects of CP violation in electroweak baryogenesis
}

\author{
Kaori Fuyuto* ${ }^{\dagger}$ \\ Department of Physics, Saga University, Saga 840-8502 Japan \\ E-mail: ss6206@cc.saga-u.ac.jp
}

\begin{abstract}
Aspects of CP-violating effects on electroweak baryogenesis in the extended Standard Model are discussed, where an extra Higgs doublet, a singlet and electroweak-interacting fermions newly exist. It is found that electric dipole moment (EDM) of the electron plays a strong role in verifying the scenario if it contains only a relevant $\mathrm{CP}$ phase. In contrast, if the other irrelevant $\mathrm{CP}$ phase interferes in the model, parameter regions where the electron EDM vanishes appear due to cancellation between CP phases. However, even in such a case, Higgs physics and the other EDMs help to examine the region.
\end{abstract}

The 11th International Workshop Dark Side of the Universe 2015

14-18 December 2015

Kyoto, Japan

* Speaker.

${ }^{\dagger}$ We thank J. Hisano and E. Senaha for collaboration. 


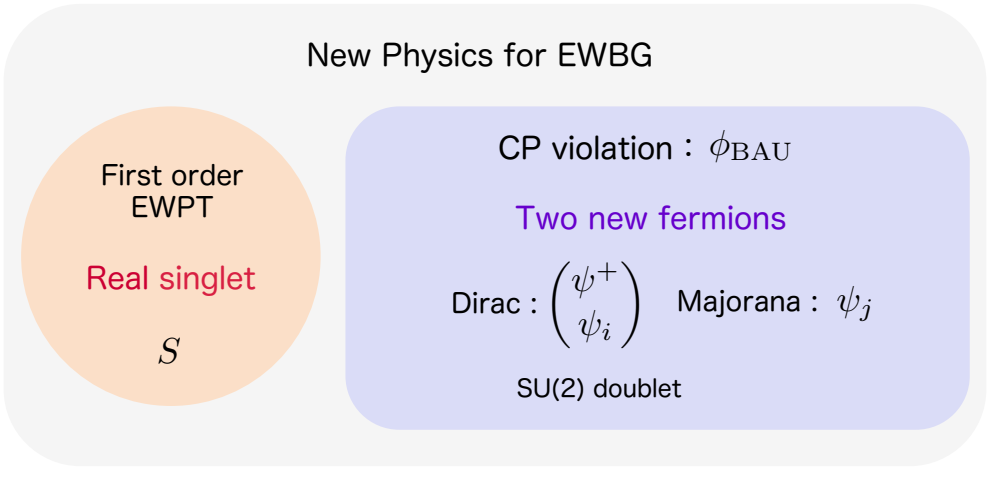

Figure 1: Our scenario for successful EWBG. Real singlet plays a role in achieving the first order EWPT, and a BAU-related CP phase $\phi_{\mathrm{BAU}}$ is supplied by interactions between two fermions, $\psi_{i}$ and $\psi_{j}$.

\section{Introduction}

In order to explain the baryon asymmetry of the Universe (BAU), $Y_{B}=(8.59 \pm 0.11) \times 10^{-11}$ [1], diverse scenarios have been invented until now. Among them, one attractive scenario is electroweak baryogengesis (EWBG) [2] in which the BAU can be created during electroweak phase transition (EWPT). Therefore, EWBG is strongly related to the Higgs physics, and it is expected that establishment of the Higgs sector plays a significant role in investigating possibility of it as existence of the Higgs boson [3] excluded the Standard Model (SM) EWBG. In addition to this, since $\mathrm{CP}$ violation is essential for the successful BAU, electric dipole moments (EDMs) can also verify the EWBG hypothesis. The verification of EWBG is promising in near future, and it is urgent issue to clarify the possibility in theoretical side.

There are two failures in the SM EWBG. One is that demand of the first order EWPT is conflict with the mass of the Higgs boson [4, 5, 6, 7], and the other is that the Kobayashi-Maskawa mechanism can not provide enough CP violation to produce the observed BAU $[8,9,10,11]$. Therefore, next candidate for successful EWBG is physics beyond the SM. Given that the current experimental results severely constrain colored particles, it is natural to discuss the possibility of EWBG in the framework where only relevant non-colored particles exist. It is known that different EWPT from that of the SM can be obtained by extending Higgs sector, and there are mainly two scenarios, i.e., (i) tree driven case, and (ii) thermal loop driven case. In the first case, tree-level potential constructs a barrier that separate two degenerate vacua, and it can be reduced to real singlet model [12, 13], effectively. The second case is that thermal loop effects of two Higgs doublet negatively contribute to the effective potential [14, 15], and non-decoupling effect is necessary for this. In both cases, the Higgs couplings may be deviated from the SM values in the region where the first order EWPT is achieved, and they become collider signals. On the other hand, although new physics usually tends to contain new $\mathrm{CP}$ violation, it is not necessarily that every $\mathrm{CP}$ phase leads to the BAU. For the verification of EWBG by the EDMs, it is needed to clarify what kind of $\mathrm{CP}$ phase is related to the BAU and EDMs.

In this talk, the possibility of EWBG in an effective model is studied, and relationship between the BAU and CP violation is also discussed. Finally, the verifiability by the EDMs and Higgs 


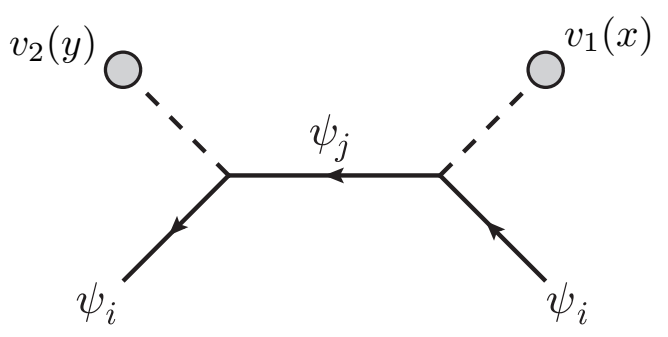

Figure 2: Diagram that describes scatterings between bubble walls and two fermions.

physics is shown. The discussion is based on [16].

\section{Relationship between the BAU and EDMs}

The concrete model we focus on is shown in Fig. 1. It is considered that, while the first order EWPT is satisfied by a real singlet, new CP violation is introduced by interactions between Dirac and Majorana fermions. In this scenario, it is possible to discuss EWPT and CP violation, separately.

In EWBG, CP violation is needed in interactions between bubble wall and particle, which leads to different transmission to bubble between particle and antiparticle. In this model, two neutral fermions $\psi_{i, j}$ have the following interactions in the basis of real mass

$$
\mathscr{L}=\frac{1}{\sqrt{2}} \bar{\psi}_{i}\left[c_{L} v_{2}(x) P_{L}+c_{R} v_{1}(x) P_{R}\right] \psi_{j}+\text { h.c, }
$$

where $c_{L}$ and $c_{R}$ are complex numbers and $v_{1,2}(x)$ are space-dependent vacuum expectation value of the Higgs. Here, the BAU-related physical CP phase becomes $\operatorname{Im}\left[c_{L} c_{R}^{*}\right]$, and the interaction can induce a digram in Fig. 2 that becomes a dominant CP-violating source for the BAU. Once we define the CP-violating source as $S_{\psi_{i}}$, it is found that final baryon number is proportional to $S_{\psi_{i}}$. $S_{\psi_{i}}$ can naively be written by

$$
S_{\psi_{i}}=C_{\mathrm{BAU}} \operatorname{Im}\left[c_{L} c_{R}^{*}\right]
$$

where $C_{\mathrm{BAU}}$ depends on parameters in the model and temperature.

Once the model has the $\mathrm{CP}$ violation, it can usually cause the EDMs. The left barr-zee diagram of Fig. 3 is induced by Eq. (2.1), where two $W$ bosons are running. It is seen that the diagram partly contains Fig. 2. As in the case of the CP-violating source term, if we write the fermion EDMs

$$
\frac{d_{f}^{W W}}{e}=C_{\mathrm{EDM}}^{W W} \operatorname{Im}\left[c_{L} c_{R}^{*}\right]
$$

it is found that there is a direct relationship between them as

$$
S_{\psi_{i}}=\frac{C_{\mathrm{BAU}}}{C_{\mathrm{EDM}}^{W W}}\left(\frac{d_{f}^{W W}}{e}\right) .
$$



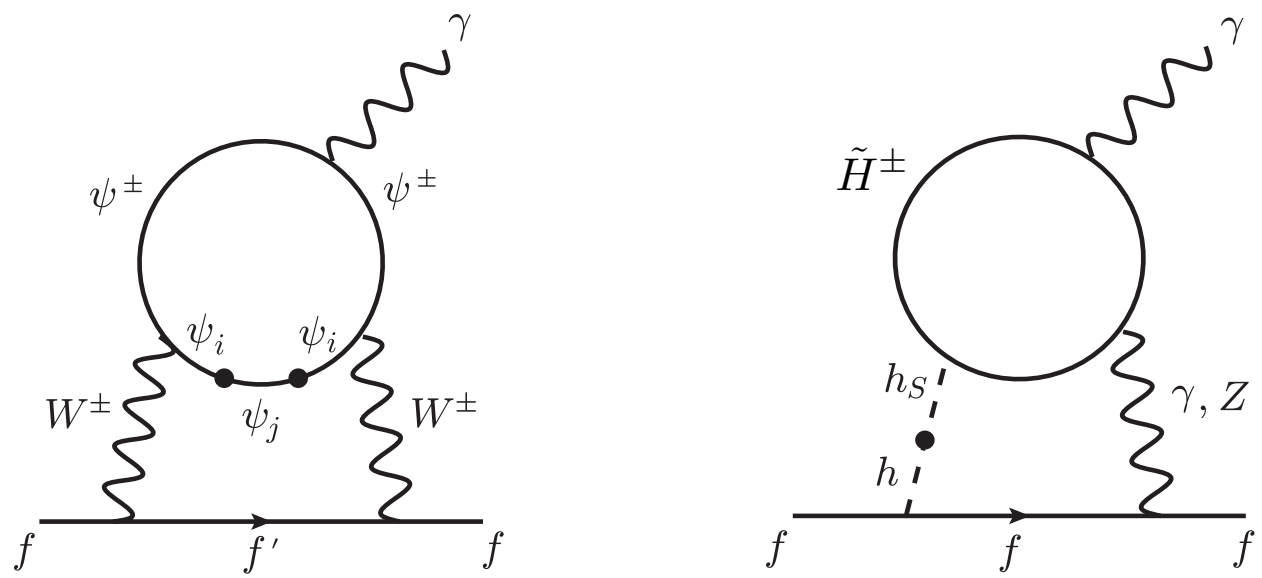

Figure 3: (Left) $W W$-mediated barr-zee diagram with the BAU-related $\mathrm{CP}$ phase. (Right) $H \gamma$ and $Z \gamma$ mediated barr-zee diagram with the BAU-unrelated $\mathrm{CP}$ phase.

In order to see behaviors of $S_{\psi_{i}}$, we replace $d_{f}^{W W}$ with the current upper limit on the electron EDM, $d_{e}^{\exp }=8.7 \times 10^{-29} e \cdot \mathrm{cm}$. Fig. 4 shows the behaviors against the masses of $\psi_{i}$ and $\psi_{j}$ where a fixed mass is $500 \mathrm{GeV}$. In this figure, model-dependent parameters in $C_{\mathrm{BAU}}$ are removed. Remarkable features in two figures are enhancements at $m_{\psi_{i}} \simeq m_{\psi_{j}}$. These enhancements lead to the successful BAU. In other words, it is only the region where the two fermions are degenerate that the observed BAU can be explained. In contrast, each large mass region differently behaves. While the electron EDM behaves as $m_{e} /\left(m_{\psi_{i}} m_{\psi_{j}}\right)$ in the heavy mass of $\psi_{j}$, it becomes $m_{e} m_{\psi_{j}} / m_{\psi_{i}}^{3}$ if $\psi_{i}$ is heavy.

\section{Results}

For numerical calculations, concrete particle contents must be determined. We consider nextto-MSSM (NMSSM)-like contents, and similar situation can be seen in $[17,18,19]$. Parameters are set in such a way as to take the limit of the real singlet model, and it is also taken that $\left|c_{L}\right|=$ $\left|c_{R}\right|=0.42$ and $\phi_{\mathrm{BAU}} \equiv \phi_{L}-\phi_{R}=225^{\circ}$. Fig. 5 shows the successful parameter region of the BAU in $\left(m_{\psi_{i}}, m_{\psi_{j}}\right)$. The black solid lines can explain the observed BAU, and the dashed lines indicate that $Y_{B} / Y_{B}^{\mathrm{obs}}=0.1$. As discussed before, the regions where fermion masses are almost degenerate are successful in explanation of the BAU. The orange region is already excluded by the electron $\mathrm{EDM}$, and the orange dashed line is prediction of $d_{e}=1.0 \times 10^{-29} e \cdot \mathrm{cm}$. Although the current experimental value excludes only some regions, it is expected that the whole possible region can be verified if $d_{e}$ reaches $10^{-29} e \cdot \mathrm{cm}$. It implies that the scenario can completely be verified by the electron EDM as long as it contains only the BAU-ralated CP phase.

However, the other $\mathrm{CP}$ phase actually exists in this model, which changes the verifiability by the electron EDM. The scenario we consider is that the real singlet cause the first order EWPT, then it has the following interactions

$$
\mathscr{L}=h_{S} \bar{\psi}^{+}\left(g^{S}+i \gamma_{5} g^{P}\right) \psi^{+},
$$

where $h_{S}$ is the real singlet and $\psi^{+}$is the charged fermion. $g^{S}$ and $g^{P}$ are given by

$$
g^{S}=|\lambda| \cos \phi_{\lambda H}, \quad g^{P}=-|\lambda| \sin \phi_{\lambda H} .
$$



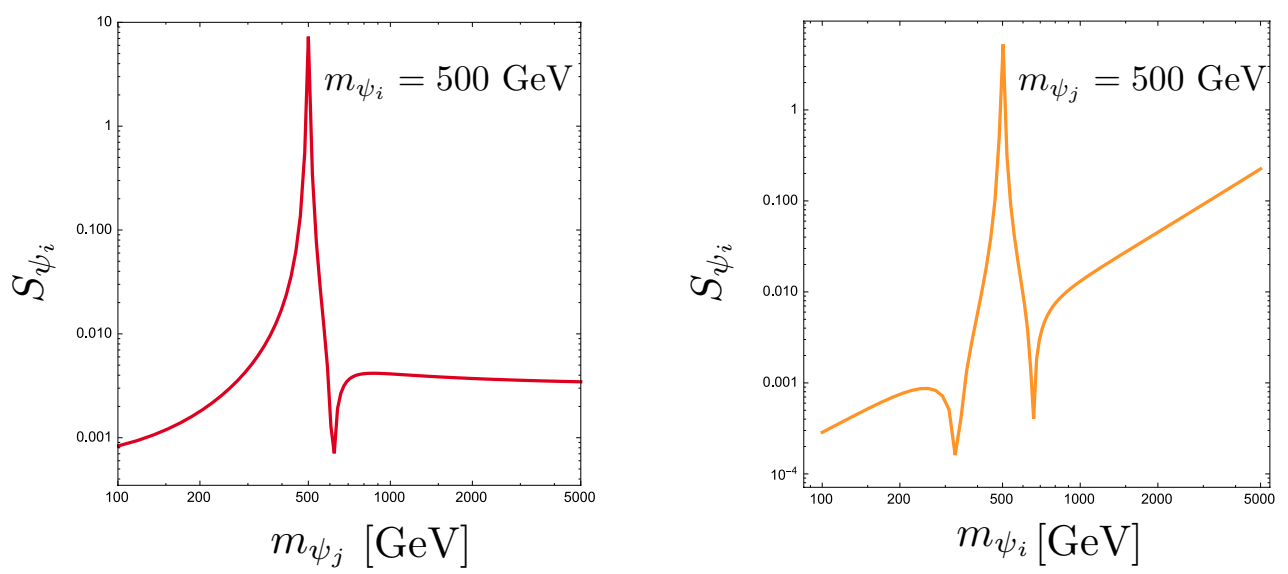

Figure 4: Behaviors of $S_{\psi_{i}}$ against the masses of $\psi_{i}$ and $\psi_{j}$. The fixed mass is $500 \mathrm{GeV}$.

The interaction introduces the new $\mathrm{CP}$ phase $\phi_{\lambda H}$, and it implies that the model have two CP phases, namely, the BAU-related and BAU-unrelated CP phase. The BAU-unrelated CP phase can induce the other contribution to the fermion EDMs as in the right diagram of Fig. 3. The barr-zee diagram is mediated by $\gamma$ and $Z$, and it surely picks up the mixing between $h$ and $h_{S}$ where $h$ is a real scalar in the $S U(2)$ doublet. Otherwise, the diagram can not connect with the SM fermions.

It turns out that total fermion EDMs can be described as $d_{e}^{\text {sum }}=d_{e}^{W W}+d_{e}^{H \gamma}+d_{e}^{Z \gamma}$, and regions of $d_{e}^{\text {sum }}=0$ appear through the cancellation between $\phi_{\mathrm{BAU}}$ and $\phi_{\lambda H}$. In such a case, the electron EDM can not play a role in the verification of the scenario. However, it should be emphasized that the $H \gamma \gamma$ loop in the $H \gamma$-mediated barr-zee diagram can contribute to signal strength of the Higgs decay to two gammas. Furthermore, the other EDMs such as neutron and proton do not vanish even if $d_{e}^{\text {sum }}=0$. Then, there is still possibility of the verification by the Higgs physics and the other EDMs.

Figure. 6 shows the contour of the electron EDM in $\left(|\lambda|, \phi_{\lambda H}\right)$ plane, where the $H Z$-mediated barr-zee diagram is not included since it is rather small compared to the $H \gamma$-mediated one. It is chosen that $\left(m_{\psi_{i}}, m_{\psi_{j}}\right)=(300,277)$ on the black line in Fig. 5 for this plot. While the red region is excluded by the electron EDM, the white region is still arrowed. The red dashed line corresponds to $d_{e}^{\text {sum }}=0$. If the electron EDM reaches $10^{-29} e \cdot \mathrm{cm}$, only the regions close to the red dashed line are left. The grey solid lines represent the signal strength of the Higgs decay to two gammas $\mu_{\gamma \gamma}$, and they are 1.1, 1.0, 0.9 and 0.8 from top to bottom. It is expected that more accurate measurement of the signal strength would narrow the allowed region as well as the electron EDM. Moreover, naive estimation shows that $d_{n} \sim d_{p} \sim \mathscr{O}(1) \times 10^{-28} e \cdot \mathrm{cm}$, and they are reachable at the future precision measurements [20, 21]. More precise estimations will be held elsewhere [22].

\section{Summary}

EWBG is the most testable scenario, and it is expected that the future collider experiments and precision measurements of the EDMs can scrutinize the possible parameter region of it. According to the current experimental results, it is considered that the colored particles do not play a role for EWBG. Therefore, it would be suitable that we consider the scenario where only the effective EW- 


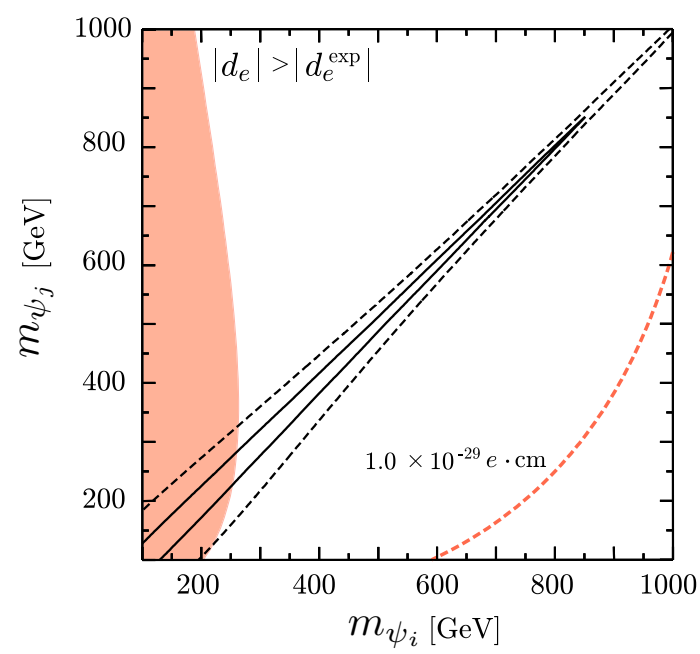

Figure 5: Contours of the BAU and electron EDM. It is chosen that $\left|c_{L}\right|=\left|c_{R}\right|=0.42$ and $\phi_{\mathrm{BAU}}=\phi_{L}-\phi_{R}=$ $225^{\circ}$. The black solid (dashed) line corresponds to $Y_{B} / Y_{B}^{\text {obs }}=1(0.1)$. While the current limit on the electron EDM excludes the orange region, the orange dashed line represents the prediction of $d_{e}=1.0 \times 10^{-29} e \cdot \mathrm{cm}$.

interacting particles exist. In addition, as the experiments improve their sensitivities, theoretical side need to clarify the possibility and identify its signals.

In this talk, we discuss the verifiability of EWBG in the concrete model by the fermion EDMs and the Higgs physics. The scenario newly introduces not only a real singlet but also two EWinteracting fermions, which can be applied to UV-complete models such as NMSSM. The former is essential for the first order EWPT, the latter supplies the BAU-related CP phase. The observed BAU can be explained in the region where two fermions are nearly degenerate. As long as only the BAU-related CP phase is present, the possible region can completely tested by the electron EDM. Though, the existence of the other BAU-unrelated CP phase slightly makes the situation complicated since it also induces the different barr-zee diagram. It results in the appearance of the region where two CP phases cancel each other out, namely, $\left|d_{e}^{\text {sum }}\right|=0$. Fortunately, even in this case, the Higgs physics can help the verification, and most of the parameter region can be surveyed. Since the other EDMs of neutron and proton have nonzero values, they also play a important role in it. Therefore, it is possible to verify the scenario by the Higgs physics and the EDMs in near future.

\section{References}

[1] P. A. R. Ade et al. [Planck Collaboration], Astron. Astrophys. 571, A16 (2014) [arXiv:1303.5076 [astro-ph.CO]].

[2] V. A. Kuzmin, V. A. Rubakov and M. E. Shaposhnikov, Phys. Lett. B 155, 36 (1985).

[3] G. Aad et al. [ATLAS Collaboration], Phys. Lett. B 716, 1 (2012); S. Chatrchyan et al. [CMS Collaboration], Phys. Lett. B 716, 30 (2012).

[4] K. Kajantie, M. Laine, K. Rummukainen and M. E. Shaposhnikov, Phys. Rev. Lett. 77 (1996) 2887 [hep-ph/9605288]. 


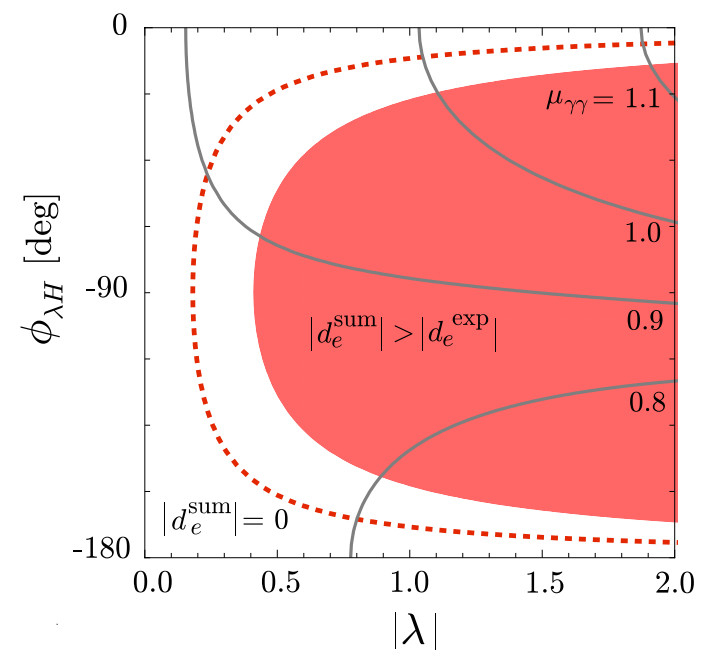

Figure 6: Contours of the electron EDM and the signal strength of the Higgs decay to two gammas in $\left(|\lambda|, \phi_{\lambda H}\right)$ plane. It is taken that $m_{\psi_{i}}=300 \mathrm{GeV}, m_{\psi_{j}}=277 \mathrm{GeV}$ and $\phi_{\mathrm{BAU}}=225^{\circ}$, which leads to the successful BAU. The red region is excluded by the electron EDM, and the red dashed line corresponds to $\left|d_{e}^{\text {sum }}\right|=0$. The grey lines are the signal strength of the Higgs, $\mu_{\gamma \gamma}=1.1,1.0,0.9$, and 0.8 from top to bottom.

[5] K. Rummukainen, M. Tsypin, K. Kajantie, M. Laine and M. E. Shaposhnikov, Nucl. Phys. B 532, 283 (1998) [hep-lat/9805013].

[6] F. Csikor, Z. Fodor and J. Heitger, Phys. Rev. Lett. 82, 21 (1999) [hep-ph/9809291].

[7] Y. Aoki, F. Csikor, Z. Fodor and A. Ukawa, Phys. Rev. D 60, 013001 (1999) [hep-lat/9901021].

[8] M. B. Gavela, P. Hernandez, J. Orloff and O. Pene, Mod. Phys. Lett. A 9, 795 (1994) [hep-ph/9312215].

[9] M. B. Gavela, P. Hernandez, J. Orloff, O. Pene and C. Quimbay, Nucl. Phys. B 430, 382 (1994) [hep-ph/9406289].

[10] P. Huet and E. Sather, Phys. Rev. D 51, 379 (1995) [hep-ph/9404302].

[11] T. Konstandin, T. Prokopec and M. G. Schmidt, Nucl. Phys. B 679, 246 (2004) [hep-ph/0309291].

[12] K. Fuyuto and E. Senaha, Phys. Rev. D 90, no. 1, 015015 (2014) [arXiv:1406.0433 [hep-ph]].

[13] S. Profumo, M. J. Ramsey-Musolf, C. L. Wainwright and P. Winslow, Phys. Rev. D 91, no. 3, 035018 (2015) [arXiv:1407.5342 [hep-ph]].

[14] S. Kanemura, Y. Okada and E. Senaha, Phys. Lett. B 606, 361 (2005) [hep-ph/0411354].

[15] K. Fuyuto and E. Senaha, Phys. Lett. B 747, 152 (2015).

[16] K. Fuyuto, J. Hisano and E. Senaha, Phys. Lett. B 755, 491 (2016) [arXiv:1510.04485 [hep-ph]].

[17] Y. Li, S. Profumo and M. Ramsey-Musolf, Phys. Lett. B 673, 95 (2009) [arXiv:0811.1987 [hep-ph]].

[18] K. Cheung, T. J. Hou, J. S. Lee and E. Senaha, Phys. Lett. B 710, 188 (2012) [arXiv:1201.3781 [hep-ph]].

[19] E. Senaha, Phys. Rev. D 88, no. 5, 055014 (2013) [arXiv:1308.3389 [hep-ph]]. 
[20] J. L. Hewett et al., arXiv:1205.2671 [hep-ex].

[21] K. Kumar, Z. T. Lu and M. J. Ramsey-Musolf, arXiv:1312.5416 [hep-ph].

[22] K. Fuyuto, J. Hisano and E. Senaha, in preparation. 